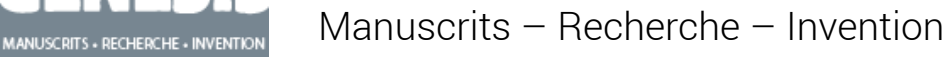

$31 \mid 2010$

Composer

\title{
Questions de faire
}

La génétique musicale in vivo... vue du côté du créateur

\section{Philippe Leroux}

\section{(2) OpenEdition}

12 Journals

Édition électronique

URL : http://journals.openedition.org/genesis/335

DOI : 10.4000/genesis.335

ISSN : 2268-1590

Éditeur:

Presses universitaires de Paris Sorbonne (PUPS), Société internationale de génétique artistique littéraire et scientifique (SIGALES)

\section{Édition imprimée}

Date de publication : 10 octobre 2010

Pagination : 55-63

ISBN : 978-2-84050-711-6

ISSN : 1167-5101

\section{Référence électronique}

Philippe Leroux, «Questions de faire », Genesis [En ligne], 31 | 2010, mis en ligne le 28 août 2012, consulté le 02 mai 2019. URL : http://journals.openedition.org/genesis/335 ; DOI : 10.4000/ genesis.335 


\section{Questions de faire La génétique musicale in vivo vue du côté du créateur}

Philippe Leroux

À l'occasion d'une session thématique du colloque "Composer au XXIe siècle », organisé par l'Observatoire international de la création musicale à la Faculté de musique de l'université de Montréal du 28 février au 3 mars 2007, trois participants à un même projet de recherche en tiraient un bilan à titre individuel et selon leurs disciplines respectives : Nicolas Donin pour la musicologie, Jacques Theureau pour l'anthropologie cognitive, Philippe Leroux pour la composition musicale. Le présent texte est directement issu de cette dernière conférence. Il nous a semblé intéressant et nécessaire, dans notre dossier thématique consacré à des démarches de recherche qui impliquent nécessairement le créateur à un degré plus ou moins fort, de donner une place au point de vue de ce dernier.

$\mathrm{D}$ OCTOBRE 2003 AU PRINTEMPS 2006, j'ai périodiquement travaillé avec Nicolas Donin, Jacques Theureau et Samuel Goldszmidt dans le cadre d'un projet à trois dimensions : recherche empirique (croisant la musicologie et l'anthropologie cognitive) sur la genèse de mon œuvre Voi(rex) (2002), conception de guides d'écoute multimédia de Voi(rex), et composition d'une nouvelle œuvre, Apocalypsis (2006). Ces presque trois années de travail commun m'ont donné une sorte de lucidité (parfois vertigineuse d'ailleurs) sur ma façon de travailler, approfondissant ainsi un mouvement réflexif commencé depuis longtemps. Cette connaissance s'est exercée à différents niveaux, que je vais essayer de vous décrire. Arrivé au terme de ces trois années, il m'a paru utile de faire le point sur ce que j'en avais personnellement tiré.

Dans la démarche de Nicolas Donin et Jacques Theureau, la composition musicale est examinée comme une activité humaine particulière, au même titre que n'importe quelle autre. J'ai particulièrement apprécié cette façon d'étudier l'activité d'un compositeur sans a priori, comme on étudierait la manière dont un paysan laboure son champ ou la fatigue du marin pêcheur. Cette attitude me paraît salutaire à une période où, en Europe particulièrement, nous avons besoin de nous désensabler d'une sorte de mystification générale concernant la notion de créateur. Ils ont compris que la création artistique était un phénomène extrêmement riche, et qu'il serait tout à fait inintelligent de chercher à l'enfermer dans quelques lois qui ne pourraient être que réductrices. Pour eux, l'activité cognitive qu'est la composition ne peut se résumer à des enchaînements de procédures. Ils ont ainsi échappé au désir un peu simpliste d'obtenir un possible « reverse engineering » qui permettrait de re-générer l'œuvre à l'identique. Je peux être tranquille : ils ne m'ont pas modélisé ! Tout au plus est-il question de modélisation analytique ou synthétique de quelques objets théoriques.

À la différence d'autres types de recherche, ils ne travaillent pas à partir des discours publics du compositeur sur sa musique, mais plutôt d'une explicitation de sa pratique compositionnelle. Ils visent à « saisir quelque chose de la création comme phénomène complexe, relevant d'une logique pratique qui ne saurait être non contradictoire ni réductible à des procédures ${ }^{1}$ ». Si vous saviez comme ce "phénomène complexe », cette «logique pratique [...] qui ne saurait être non contradictoire », m'ont rassuré quand je les ai connus. Phénomène complexe, car non réductible à quelque dispositif d'investigation que ce soit ; logique pratique, car l'aspect artisanal de l'art est loin d'être négligeable ; qui ne saurait être non contradictoire, car il me semble qu'une activité de création musicale qui se voudrait sans contradiction serait bien naïve, et risquerait de générer de piètres œuvres d'art. 
Au fil des séances de « remise en situation », où Nicolas et Jacques m'ont interrogé pas à pas sur les détails du cours même de mon activité compositionnelle, à la lecture de leurs analyses publiées régulièrement sous forme d'articles, ainsi qu'en observant ma façon de travailler lors de la composition de nouvelles œuvres, j'ai accumulé des réactions, des réflexions, des connaissances relatives à mon propre travail créateur. Pour en faire ici un bilan, je les parcourrai à travers quelques grandes catégories (dont certaines se recouvrent nécessairement) : ce que je savais sans l'avoir nommé, ce que je découvre, ce que je souhaite approfondir, ce que je peux revendiquer, etc.

\section{Ce que je savais sans l'avoir nommé}

De mon point de vue, un des premiers apports de cette étude a été la mise en lumière d'un certain nombre de constituants du travail de composition, que je connaissais sans les avoir pour autant nommés.

Ainsi de l'importance des éléments implicites dans l'activité à chaque instant, qui sont tellement familiers qu'ils n'apparaissent dans aucun plan ni aucune note. Ces éléments sont pourtant, de façon cachée, à la base de nombreuses décisions importantes qui s'effectuent instantanément. Ils sont un des éléments principaux de ce qu'on appelle la conscience préréflexive, l'autre part étant constituée de ce qu'on nomme la conscience, au sens usuel du terme. Par exemple, mon aversion pour la pure symétrie implique que dans le quatrième mouvement de Voi(rex), la déconstruction des blocs gigognes structurant cette section se fasse nécessairement d'une façon dissymétrique à la période de construction, et cela de façon évidente, sans qu'il soit besoin de le noter, ni même de l'énoncer lors des entretiens. C'est souvent de la confrontation entre ces éléments implicites et d'autres définis à l'avance que naît l'écriture musicale. Comme autres éléments implicites, on pourrait citer ce que je sais ne pas vouloir écrire : ce qui me ferait trop me rapprocher du style précis d'une période de l'histoire de la musique, ou d'un autre compositeur, ou encore ce qui contreviendrait à mon besoin que les événements s'induisent les uns les autres. Dans ce domaine de l'implicite, je différencierai l'implicite fondamental qui est valable pour toutes les œuvres, de l'implicite local lié à une pièce en particulier. Pour ce dernier, je citerai la relation évidente que j'établis entre les «blocs gigognes » du quatrième mouvement de Voi(rex) et la nécessité d'une forte densité.

J'en arrive parfois à croire que ce qui a laissé des traces, que ce soit dans les plans et esquisses ou dans les entretiens de remémoration effectués avec Nicolas et Jacques, n'est que la partie émergée d'un iceberg, la plus grande part des décisions appartenant à la conscience préréflexive et se prenant de façon quasiment inconsciente parce qu'implicite. On peut d'ailleurs imaginer qu'un certain nombre de procédés, ne provenant pas initialement du domaine des éléments implicites, mais que le travail de recherche entrepris a permis de nommer, basculeront vers l'implicite une fois identifiés (par exemple une problématique qui était difficile à cerner ou à définir). Car dans ce domaine se trouvent bien sûr des attitudes qui n'ont jamais effleuré la surface de la conscience, mais également d'autres, qui après avoir été identifiées et conscientisées, n'offrent plus ou peu d'intérêt conscient et basculent dans le domaine de l'implicite.

La notion d' « idée » reprise par Nicolas et Jacques à un commentaire de Kant par Deleuze ${ }^{2}$ me semble assez proche de l'emploi que j'en fais. Elle présente trois moments : " indéterminée dans son objet, déterminable par rapport aux objets de l'expérience, portant l'idéal d'une détermination infinie par rapport aux concepts de l'entendement ${ }^{3} »$. L'indétermination liée à ce premier moment n'est plus alors considérée comme un manque dans la connaissance que l'on a de la future œuvre, mais elle est un élément positif qui se situe dans un potentiel et un horizon de possibilités. L'indéterminé, outil puissant au service de la construction artistique, s'objective à travers cette dernière.

1. Nicolas Donin et Jacques Theureau, «Voi(rex) de Philippe Leroux, éléments d'une genèse. Reconstitution analytique du processus créateur d'une œuvre récemment créée », Dissonanz/Dissonance, ${ }^{\circ}$ 90, juin 2005, p. 7.

2. Nicolas Donin et Jacques Theureau, « La composition d'un mouvement de Voi(rex), de son idée formelle à sa structure », L'Inouï, revue de l'Ircam, n 2, 2006, p. 62-85. 
L'idée possède son identité propre qui la différencie des autres, elle se présente sous forme symbolique, elle est simple, encore vague et possède un fort potentiel. Elle s'inscrit sous forme de dessins annotés ou non, de commentaires littéraires ou de symboles musicaux. Chez moi, la naissance de l'idée est un instant extrêmement important de la composition, lié en général à une émotion dont la cause peut être variable. Ce moment est suivi par une première notation assez primaire, intermédiaire entre ce qui relèverait du discours privé et du langage public. Suivent un classement des différentes idées, un report sur un ou des plans, une organisation de ces idées entre elles et leur réalisation dans le cadre de la partition. Au fur et à mesure du travail compositionnel, elles se précisent de plus en plus et s'épuisent dans une partition donnée, pour éventuellement réapparaître dans une autre.

Au-delà de l'idée sonore, en ce qui concerne la structure, on différenciera l'idée formelle de la prédétermination formelle 4 . L'idée formelle possède un potentiel de significations beaucoup plus important que la prédétermination. Celle-ci est trop précomposée pour constituer une idée, elle est déjà disponible pour la composition et ne suppose pas une découverte. Ce n'est pas elle qui, dans le temps de la composition, porte l'émotion ; c'est plutôt sa réalisation. Elle n'est pas appelée non plus à perdurer dans une future pièce.

Une autre caractéristique importante de ma façon de travailler est de séparer nettement l'étape de préparation des éléments servant à la composition, de celle de l'écriture de la partition : «Alors que la préparation permet de réaliser des associations entre toutes sortes d'idées musicales afin de définir la physionomie globale de chaque mouvement et d'anticiper les relations entre mouvements, l'écriture [...] implique [...] une attention constante, appuyée sur les documents issus de la préparation, à la réalisation d'un tissu de liens (prédéfinis ou non) entre un grand nombre de passages de la partition ${ }^{5}$. C'est dans la phase de préparation que naissent les plans, qui ne sont encore à ce moment que des supports de possibles à actualiser, relier et compléter au fur et à mesure de la composition de l'œuvre. J'ai toujours pensé que composer une pièce était comme bâtir un édifice : on définit des matériaux, on imagine des relations entre eux, on planifie, puis on amène ces matériaux sur place et on commence à construire. Il n'est pas question, quand on travaille à l'achèvement d'une tour, d'aller couper un arbre dans la forêt, puis de le faire sécher, parce qu'il manque un chevron. Il en va de même pour la composition (bien que cela dépende évidemment de chacun et de ce qu'on entend par matériau compositionnel) : il n'est plus question au milieu d'une œuvre de remettre en cause les matériaux morphologiques, harmoniques ou autres. On peut à la rigueur les compléter, mais les « ré-inventer » complètement ferait voler en éclats toute la cohérence de l'œuvre (...à moins bien sûr que ce procédé ne fasse partie du projet initial).

Après l'étape de la préparation vient celle de l'écriture à proprement parler. Le temps de l'écriture est pour moi le dépliement de ces moments d'intense écoute intérieure et d'extrême concentration sur les choix fondamentaux du déroulement temporel et sur l'ajustement de chaque élément. L'écriture de la partition se fait linéairement, dans la continuité musicale, du début à la fin de la pièce. Elle s'appuie sur les documents issus de la préparation. Elle tente de réaliser un tissu de liens (prédéfinis ou non) entre les différents passages qui la constituent. Elle est parsemée de relectures multiples, qui sont autant de « relectures stratégiques 6 »- stratégiques parce que intervenant à des moments très précis de l'écriture - au fur et à mesure de l'avancée dans la partition. Le premier type de relecture se fait avant la phase d'écriture de chaque mouvement, et concerne non pas la partition elle-même, mais les brouillons, les plans et toutes les notes concernant ce mouvement particulier. Le deuxième type de lecture porte sur ce qui a déjà été écrit sur la partition. Il s'agit d'écoutes et de réécoutes de la partition

3. Gilles Deleuze, Différence et répétition, Paris, Presses universitaires de France, 1968, p. 220 (commenté dans N. Donin et J. Theureau, « La composition d'un mouvement de Voi(rex)... », art. cit., p. 65).

4. N. Donin et J. Theureau, « La composition d'un mouvement de Voi(rex)... », art. cit., p. 63.

5. N. Donin et J. Theureau, « Le sentiment de la forme. Analyse génétique et cognitive de la composition d'un mouvement d'Apocalypsis par Philippe Leroux », Musurgia. Analyse et pratique musicales, à paraître en 2011.

6. N. Donin et J. Theureau, « L'activité de composition musicale comme exploitation/construction de situations. Une anthropologie cognitive du travail de Philippe Leroux », Intellectica, vol. XLVIII, n 1-2, 2008, p. $175-205$. 
dans sa continuité, intégrant dans l'audition intérieure aussi bien ce qui relève du domaine instrumental que de l'électronique. Ces réécoutes intérieures reprennent à chaque fois la lecture au début du mouvement concerné voire au début de l'œuvre. De telles lectures successives mettent à l'épreuve les matériaux et leurs mises en relation. Elles seules permettent de codéterminer les différents éléments en situation. Tout ce qui avait été imaginé dans la préparation est maintenant pris dans une dynamique susceptible d'altérer à tout moment certaines des relations et des contraintes prévues.

Il résulte parfois de cette mise à l'épreuve de la préparation par des réécoutes intérieures successives un écart important entre ce qui était préparé et ce qui est finalement écrit. Ce qui avait été imaginé n'est pas en phase avec la réalité sonore et musicale de la pièce. Cet écart, assumé et accepté, amène un recadrage de l'idée première. Sans que cela ne signifie un renoncement à celle-ci, les déviations qui apportent quelque chose sont conservées. Ce qui est écrit dans la suite de l'œuvre tient compte de cet écart, et ce qui était prévu initialement doit s'ajuster en fonction de la déformation subie. Ce recadrage peut porter sur des proportions temporelles, des hauteurs, des densités ou tout autre constituant musical. Il peut porter sur une courte portion temporelle comme sur toute la suite de la pièce. Le projet général de l'œuvre s'actualise au fur et à mesure de ces confrontations entre le prévu et le réel.

\section{Ce que je découvre}

Le travail de recherche collaboratif m'a amené également à faire des découvertes, par la prise de conscience d'aspects inaperçus de mon propre artisanat.

La première phase dans mon travail de composition consiste à préparer un ensemble de possibles, mais aussi, à susciter des problèmes potentiels de composition à résoudre. Problèmes, au sens où la composition musicale fait partie du domaine de la recherche au sens large, mais aussi dans un sens plus pédagogique : d'une pédagogie pour soi-même. Dans tout mon travail, on trouve cette attitude à confronter la réalité sonore à des contraintes qui lui sont extérieures. Cela est valable, par exemple, pour une certaine façon de mettre en perspective écriture instrumentale et utilisation d'éléments électroacoustiques. Dans le cadre qui nous occupe, il s'agit plus de susciter un problème de composition, puis de tenter de le résoudre afin de trouver des façons de faire non familières ni habituelles. Cela me fait accéder à un type d'écriture musicale dont je n'aurais pas eu l'idée par moi-même. Je n'ai pas envie que ma musique ne soit que la réalisation de mes fantasmes musicaux. Rien ne serait plus triste. Je souhaite que les sons, mais aussi les idées, les concepts et les structures me mettent en question et me permettent de déboucher sur du nouveau, naissant de ces situations nouvelles. À moi de savoir si, par la suite, je souhaite intégrer cela à une manière de faire plus générale qui participe de mon style.

On évoquera aussi, dans une interprétation plus ludique, le ravissement éprouvé à résoudre ces problèmes - ce principe de difficulté gratuite qui met en valeur le plaisir ressenti à résoudre une difficulté et à se plier à des conventions préétablies, ce que Roger Caillois nommait le ludus ${ }^{7}$.

Certains des problèmes de composition seront résolus dans cette première phase. Mais c'est celle de l'écriture qui devra en résoudre le plus grand nombre, au fur et à mesure de l'avancée de la partition. La phase de préparation n'a pas particulièrement vocation à résoudre des problèmes globaux de forme, alors que celle de l'écriture se concentrerait uniquement sur des problèmes locaux : dans ma méthode, la forme globale peut résulter, pour une part relativement importante, de la période d'écriture.

Ces problèmes peuvent être résolus, déplacés, contournés ou dissous. Ils sont résolus lorsque ce qui a été prévu dans la préparation a été respecté ; déplacés, quand ils sont finalement solutionnés, mais au prix d'une transformation de l'idée (alors interprétée d'une façon différente de celle imaginée au début) ; contournés, quand la problématique en question ne peut finalement déboucher au sein de l'œuvre en cours, mais se trouve reportée vers une œuvre ultérieure ; dissous enfin, lorsque toute tentative de résoudre un de ces problèmes est

7. Je me suis référé à cette notion (exposée dans Les Jeux et les hommes : le masque et le vertige, Paris, Gallimard, 1958) dans mon article : « La composition : jouer ou mourir. Quels sont les rapports que peuvent entretenir le jeu et la composition musicale ? ", Dissonanz/Dissonance, $\mathrm{n}^{\circ} 82$, août 2003, p. 20-25. 
définitivement abandonnée, parce que ayant perdu de son intérêt dans le fil de l'écriture de l'œuvre.

De là, j'ai réalisé que la phase d'écriture de la partition se déroulait simultanément sur deux niveaux. Le premier consiste dans la mise en place de ce qui a été prévu lors de l'élaboration des plans de l'œuvre. À cette mise en place s'ajoutent nécessairement quelques changements ou quelques événements non prévus, d'où l'existence d'un deuxième niveau qui caractérise quant à lui les diverses réactions que suscitent tout au long de l'œuvre ces nouveaux éléments. Comme pour l'angle naissant des deux branches formant un triangle, une microvariation au début de la pièce peut devenir un élément très important d'une partie ultérieure.

S'écrivent donc en parallèle deux histoires simultanées : l'une, à partir des plans et des matériaux préparés en amont, l'autre, ne réagissant qu'aux éléments imprévus déjà couchés sur le papier. Je parlerais volontiers pour la première d'écriture à caractère extrinsèque, car elle renvoie à un ensemble d'éléments prédéterminés lors de la phase de préparation, qui de ce fait, sont extérieurs au temps de rédaction de la partition. La seconde me semble de caractère intrinsèque puisqu'elle s'appuie uniquement sur les relations existant entre les événements musicaux tels qu'ils existent de façon autonome dans le temps de l'œuvre musicale.

\section{Ce que je souhaite approfondir}

Avoir une vision plus claire de mon comportement compositionnel me conduit à souhaiter approfondir certaines questions, ceci dans un but autopédagogique. Par exemple, que dois-je penser de la dissolution ou du contournement des problèmes évoqués plus haut ? Si cela ne pose guère de questions dans une pièce particulière, il me semble cependant y entrevoir globalement un manque d'adéquation entre une préparation de situations de compositions et leurs possibilités de réalisation. Il est vrai qu'au fur et à mesure de mon expérience compositionnelle et de la connaissance de ma méthode de travail, l'équilibre entre la mise en place préalable de structures et de matériaux, et la possibilité de leurs réalisations dans le travail d'écriture de la partition s'est beaucoup transformé et, de mon point de vue, amélioré. Dans mes premiers travaux, autour des années 1975, la distance entre les deux était considérable, au point que la préparation n'avait souvent comme effet que d'avoir exploré les limites d'un terrain compositionnel, ou même, dans les cas extrêmes, d'avoir inventorié les conduites musicales à éviter ; quant à l'écriture, elle devait presque à elle seule supporter le poids de la construction aussi bien que celui de la réalisation proprement dite. Depuis, ces deux moments fondamentaux de la composition se sont développés dans une synergie toujours plus grande. Ce souci d'équilibre ne m'est pas réellement spécifique, mais il n'est pas partagé par tous les compositeurs. Pour certains, le fait de préparer d'importantes constructions théoriques n'ayant que peu de rapport avec la réalisation qui suit, et encore moins avec le résultat sonore final, ne pose pas de problème particulier. Quoi qu'il en soit, je vois donc dans ces dissolutions et ces contournements de problèmes quelque chose à améliorer dans ma façon de faire.

Il en est de même des contradictions relevées lors des entretiens avec Nicolas et Jacques au sujet de la répartition harmonique du quatrième mouvement de Voi(rex), qui aurait pu servir plus efficacement la problématique générale de ce mouvement. Dans celui-ci, les structures formelles et de rythme harmonique sont totalement dissociées alors qu'il aurait pu être intéressant de chercher à les coordonner. Observer de près ce genre de contradiction permet d'en tirer d'excellentes leçons pour les œuvres suivantes. Je pense d'ailleurs en avoir profité lors de la composition d'Apocalypsis.

La question du silence, au cœur de ce quatrième mouvement de Voi(rex), a révélé elle aussi une relative inadéquation, une insatisfaction en tout cas, entre le projet initial et sa concrétisation musicale. Pointer cela m'a permis de résoudre le même problème lors de sa reprise dans Apocalypsis, où le silence est devenu l'élément essentiel du principal mouvement.

Prendre conscience de la lecture partielle que j'avais faite de la problématique des «blocs gigognes » dans Voi(rex) m'a été aussi fort utile. De cette idée, inscrite sous forme de schémas dans de nombreuses esquisses, je n'avais tout d'abord voulu voir que l'aspect horizontal (temporel), sans prendre en charge, dans la phase de réalisation, les implications d'une lecture graphique 
verticale de ma représentation de ces blocs. Cela avait ultérieurement constitué un obstacle à la compréhension que Nicolas et Jacques essayaient d'avoir de la composition de ce mouvement ; leur réaction interloquée nous avait collectivement amenés à éclaircir les implicites de nos façons divergentes d'interpréter un tel schéma dans mes esquisses. Retravaillant sur l'idée de «blocs gigognes » dans Apocalypsis, j'en ai, cette fois, exploré aussi le côté vertical et polyphonique. Ma vision en devient évidemment plus riche et complexe.

En somme, j'ai parfois détourné le travail de recherche en une sorte de cours de composition que je désespérais d'avoir jamais.

\section{Ce que je peux revendiquer}

Avoir une meilleure compréhension de mes conduites musicales m'amène à discerner, parmi elles, ce que je peux et veux plus particulièrement revendiquer.

La question des relectures stratégiques, déjà évoquée, en est un exemple. Le projet de l'œuvre s'actualise à mesure de l'écoute intérieure que j'en ai. Les choix d'agencements des matériaux qui ont été opérés dans la phase de préparation sont mis à l'épreuve d'une écoute continue. Durant le temps même de cette écoute singulière, ces matériaux sont mis en relation de façon instantanée, ce qui amène parfois des changements par rapport aux prévisions, ou bien conduit à ajouter des éléments dans le fil du discours musical. Cette écoute active cherche à consumer ou à épuiser les matériaux choisis. Elle veut en extraire le suc, et cherche à les mettre au maximum en relation les uns avec les autres. Ce besoin d'appliquer au déroulement musical une attention constante dans la continuité d'une écoute s'explique par le fait que j'écrive en général de la première à la dernière page, gommant les esquisses et corrigeant au fur et à mesure. Je ne reviens que très rarement en arrière, et ceci uniquement dans le dessein de compléter ou d'affiner tel passage, sans transformer en profondeur ni effacer de longues sections. Il m'est indispensable de sentir une unité qui serait peut-être celle d'un geste formel, constitué d'une multitude d'enchaînements sonores déductifs. Je ne peux m'empêcher de penser à l'unique trait de pinceau, cœur de l'enseignement du peintre chinois Shitao. Dans sa théorie, le trait est à la fois forme et mouvement, volume et valeur de teinte. Il forme en soi une unité vivante, une sorte de traduction visuelle de ce concept essentiel de la philosophie taoïste qu'est le Souffle unificateur. Pour Shitao, « tout réside en l'homme, par le libre élan du seul pinceau, de la seule encre 8 ! ». Pour moi, le pinceau serait l'écoute intérieure active évoquée précédemment, de laquelle peut naître l'unique trait, c'est-à-dire ce qui assure l'unité de l'œuvre dans la diversité de ses constituants. L'encre serait plutôt le matériau soigneusement choisi dans un travail réalisé en amont. C'est comme si la place choisie au préalable pour chaque élément devait passer au crible de la perception intérieure.

Comme l'écrivent Nicolas et Jacques, il importe d' « expliciter ce qui, dans le processus de composition comme dans l'œuvre achevée, ne se laisse pas réduire au dépliement d'une musique prédéfinie 9 ». Je ne fais confiance à la réflexion préécriture que si elle est vérifiée et enrichie par la perception. On ne peut évidemment pas dire qu'une œuvre d'art soit véritablement vivante, mais à défaut de «vivant», il me semble travailler sur du «mouvant », avec toutes les surprises qui peuvent en découler. Un être humain ne peut ni savoir ni décréter ce qu'il fera dans sa vie. Il lui faut la vivre, et parfois s'adapter en fonction du contexte. Il y a là une certaine analogie avec l'œuvre musicale : on ne peut tout y prévoir à l'avance, sans passer par le vécu musical qui ne peut se faire que dans le temps. La musique est un art du temps. Vouloir l'imaginer entièrement dans un temps figé et immobile risque de conduire à des approximations grossières. De cet équilibre entre préparation hors-temps et écoute intérieure en-temps peut naître l'identité réelle de la pièce.

Cet unique trait de pinceau est, en quelque sorte chez Shitao, ce qui unifie dans sa peinture paysagère des milieux naturels aussi différents que la mer ou la montagne. L'écoute intérieure en-temps permet elle aussi d'unifier

8. Les Propos sur la peinture du moine Citrouille-Amère : traduction et commentaire de Shitao, éd. Pierre Ryckmans, Paris, Plon, 2007.

9. N. Donin et J. Theureau, «La composition d'un mouvement de Voi(rex)... », art. cit., p. 63. 
des éléments très disparates. L'attitude traditionnelle, en composition, est de créer tout d'abord un noyau initial à partir duquel sont générés tous les autres éléments de la pièce. Il est cependant possible de procéder d'une manière différente, et à l'inverse, de tout ramener à un noyau, en trouvant ce qui peut unir une suite d'éléments hétérogènes. On pourrait qualifier cette façon de faire d' « écologique10 », en ceci qu'elle permet d'utiliser et de recycler des éléments qui a priori ont peu ou pas de rapports entre eux. Peut-être correspond-elle à une époque où se fait sentir un pressant et vital besoin de synthèse.

Nicolas et Jacques ont montré que dans le troisième mouvement de Voi(rex), les aplats d'accords relient toutes les idées entre elles, aussi différentes soient-elles. Ces aplats sont le noyau unifiant, l'unique trait de pinceau. C'est ce noyau qui permet de mettre en réseau tous les éléments constitutifs de l'œuvre. Par ailleurs, l'utilisation de processus de transformation des éléments participe elle aussi de la gestion de l'hétérogène par l'établissement de fortes relations entre les différents événements musicaux.

\section{Ce que j'utilise directement dans Apocalypsis}

La partie multimédia du projet avec l'équipe Analyse des pratiques musicales a elle-même produit des outils (applications informatiques) que j'ai souhaité intégrer dans la composition de la nouvelle œuvre mise en chantier au cours de la même période, Apocalypsis. Cette œuvre s'inspirant directement de la genèse de Voi(rex) - réservoir d'idées, de matériaux et de problèmes à reprendre ou à représenter -, il me semblait naturel d'utiliser ces dispositifs explicatifs et analytiques, susceptibles d'en rendre compte. C'est le cas pour une application permettant de visualiser la spatialisation du premier mouvement de Voi(rex). Les données spatiales qui en sont issues ont été converties en données de hauteurs, afin que ce qui était trajectoire du son dans l'espace devienne mouvement mélodique. Un outil portant sur le deuxième mouvement, et permettant d'associer le graphisme des lettres avec des profils mélodiques, m'a permis de recomposer un autre sens musical et littéraire en interpolant lettres et fragments mélodiques. Une autre application analyse la notion de « modèle du modèle » dans le troisième mouvement, par la mise en lumière des relations existantes entre les accords instrumentaux, leur utilisation par l'électronique, leurs traitements, la simulation harmonique de ces traitements et leur réécriture instrumentale. Cet outil m'a donné un cadre de mise en relation entre écriture instrumentale et électronique que j'ai utilisé tel quel dans le cinquième mouvement d'Apocalypsis.

Le fait d'avoir isolé, mis en relief ou discrétisé les différentes étapes de la composition m'a incité à utiliser celles-ci comme éléments structurels. C'est le cas dans le troisième mouvement d'Apocalypsis qui met musicalement en scène, et de façon chronologique, l'enregistrement de la voix de la chanteuse déclamant les poèmes de Lin Delpierre dans des résonateurs tels que des gongs ou des tam-tams, une sélection de fragments de ces enregistrements, leur analyse spectrale puis la conversion des résultats d'analyse en données symboliques, une nouvelle séance d'enregistrement d'instruments jouant des accords issus de ces données, une sélection de fragments de cet enregistrement suivi d'un premier traitement des éléments de cette sélection.

La transcription des entretiens de remise en situation m'a servi de matériau littéraire pour la nouvelle pièce. J'en ai extrait un ensemble de phrases qui me semblaient pertinentes d'un point de vue poétique, dramatique, phonétique et sémantique, et les ai réparties selon les différents mouvements. Cette façon de faire donne à la pièce ce caractère autoréférentiel qu'on peut parfois trouver dans certaines pièces de l'Ars Nova au XIVe siècle dans lesquelles le texte explique la façon même dont il faut lire la partition ou dont celle-ci est construite.

Avoir revécu, lors des entretiens, l'abandon du nombre de mouvements de Voi(rex) prévus initialement (huit étaient prévus, l'un a été abandonné tandis que trois autres étaient ramassés en un seul) m'a sans doute incité à souhaiter les réintroduire dans la nouvelle œuvre. C'est le cas pour le second mouvement d'Apocalypsis : il reprend l'idée de ce qui aurait dû être

10. Ce que j'entends par le mot écologique est bien sûr différent du sens que lui conférait Grisey dans ses textes de la fin des années soixante-dix (voir par exemple Gérard Grisey, Écrits ou l'invention de la musique spectrale, éd. Guy Lelong, Paris, Éditions MF, 2008, p. 28). 
le quatrième mouvement de Voi(rex), et qui consiste en une énumération pure et simple de toutes les idées sonores et structurelles de la première pièce. Y est associé le défi d'imaginer comment rendre compte, de façon sonore, d'une structure abstraite. Autre rebond d'une pièce à l'autre : les « intermèdes » prévus dans Voi(rex) ont finalement vu le jour dans la nouvelle œuvre, toujours associés aux accords pressentis initiaux.

Il est vrai qu'après l'écriture d'une partition, certains mécanismes ont tendance à être refoulés, et tout ou partie du comment d'une composition à être oublié, car finalement seul le résultat compte. Ce travail d'analyse m'a amené à remettre à plat un certain nombre de comportements compositionnels, et à les explorer davantage dans le cadre d'une nouvelle composition.

\section{Ce qui a pu influencer inconsciemment mon travail de composition dans Apocalypsis}

Il me semble légitime de se demander dans quelle mesure ce travail de recherche a influencé inconsciemment la composition de la nouvelle pièce. La partition finale de Voi(rex) n'ayant pas strictement correspondu au schéma préétabli, on peut se poser la question de savoir si la présence du regard extérieur de Nicolas et Jacques ne m'a pas encouragé à achever à tout prix Apocalypsis selon le plan initial. Il me semble important de préciser que Voi(rex) avait été de ce point de vue une sorte d'exception : ainsi, par exemple, De la vitesse (l'œuvre immédiatement précédente), possède le nombre de mouvements inscrits dans le plan. S'il est certain que leur présence accompagnante ne pouvait que me donner envie de réaliser l'œuvre au plus près de celuici, cela n'est en aucun cas exceptionnel.

Il est vrai également que pendant la composition d'Apocalypsis, une ou deux remarques de Nicolas et Jacques m'ont amené à trouver une solution concernant certains passages plus rapidement que je ne l'aurais fait seul. Mais, outre que cela ne s'est produit qu'une ou deux fois, il est probable que la solution trouvée par mes seules forces aurait été la même, ou, du moins, très proche.
En dehors du fait de me prouver l'intérêt de tenir un agenda de composition11, pratique dont l'importance pédagogique est réelle - je le conseille maintenant à mes étudiants avancés pour sortir d'une certaine routine -, l'étude menée pendant ces trois années aura porté différents types de fruits en ce qui me concerne. Le principal est certainement une meilleure connaissance de soi-même. Les données produites par cette recherche apportent une aide précieuse dans la réalisation de retours réflexifs sur mon activité de composition. Ils permettent de nommer des façons de faire que je connaissais déjà et d'en découvrir d'autres. Certaines que je souhaite approfondir, ou corriger, d'autres revendiquer en tant que style propre à mon activité, d'autres encore que je peux utiliser directement dans la composition comme un matériau conceptuel, technique ou symbolique.

Pour finir, je dirais que tout a été possible parce que j'ai été associé dès le début au travail de recherche. Mon rôle a été actif dans la construction des données sur cette activité. Le projet a été partagé - même si l'essentiel de ma réflexion a porté sur la composition de ma nouvelle œuvre - ainsi que les techniques mises en œuvre pour cette recherche, notamment la méthode de remise en situation de composition. Nous sommes ici à l'opposé d'une situation réifiante où je n'aurais été qu'un objet passif dont le comportement aurait été examiné par des chercheurs le plus extérieurs possible, comme si c'était là la meilleure façon de garantir l'objectivité des résultats. Ce sont des rapports entre des personnes vivantes, moi et les chercheurs, et une expérience vécue par chacun de nous qui ont permis la construction de cette exploration anthropologique, musicologique, technologique et compositionnelle, dont, en ce qui me concerne, je suis encore loin d'avoir décrypté tous les résultats.

11. Cette demande des chercheurs pour le suivi in vivo de la composition d'Apocalypsis répondait, pour eux, au besoin d'un guide d'entretien sommaire court-circuitant le rétrospectivisme téléologique, inévitable lors d'un entretien portant sur plusieurs semaines passées de travail d'écriture. Même si j'en voyais l'utilité pour le projet de recherche, je n'étais, au début, pas du tout sûr de pouvoir adopter une pratique qui m'avait été jusqu'alors étrangère. 
Philippe Leroux, compositeur français né en 1959, étudie avec Ivo Malec, Claude Ballif, Pierre Schaeffer et Guy Reibel, ainsi qu'Olivier Messiaen, Franco Donatoni, Betsy Jolas, Jean-Claude Eloy et Iannis Xenakis. Auteur de nombreuses œuvres instrumentales, vocales et électroacoustiques (publiées aux éditions Gérard Billaudot), il est également professeur de composition musicale, notamment à l'Ircam (Cursus de composition assistée par ordinateur, 2001-2006) et, depuis 2009, à l'université de Montréal.

Philippe Leroux, continuo@wanadoo.fr

\section{Résumés \\ Questions de faire. La génétique musicale in vivo vue du côté du créateur}

Si les conséquences de la « génétique in vivo » restent à préciser aux plans méthodologique et épistémologique, il est d'ores et déjà possible d'amorcer la réflexion sur un aspect inédit de ce type d'étude : le créateur étant nécessairement impliqué (plus ou moins étroitement) dans le projet de recherche, il se forme un point de vue spécifique sur ce dernier et pourrait même, dans certains cas, y contribuer en parallèle avec des chercheurs. Philippe Leroux propose ici une reformulation dans ses propres termes du projet de recherche de Nicolas Donin et Jacques Theureau auquel il a participé, et il parcourt les différents types de savoir ou de prise de conscience occasionnés par la série d' « entretiens de remise en situation de composition » auxquels il s'est livré pendant une période de trois années (2003-2006) relativement à deux de ses principales œuvres de cette période.

If on a methodological and epistemological level the consequences of "live genetics" are still to be specified, it is already possible to undertake a reflection about an unobserved aspect of this type of study: as the creator is necessarily more or less implicated in the research project, this creator develops a specific point of view towards this project and in certain cases may even contribute to the project at the same time as the researchers. Philippe Leroux suggests a new formulation in his own words of Nicolas Donin and Jacques Theureau's research project in which he participated. He then peruses the various types of knowledge or awareness produced by the "composition situation scenario interviews" in which he cooperated for three years (2003-2006) concerning two of his main works of that period.

Obgleich es die Konsequenzen der « Genetik in vivo » auf methodologischer und epistemologischer Ebene noch genauer zu fassen gilt, ist es schon jetzt möglich, über einen neuartigen Aspekt dieser Forschungsrichtung nachzudenken: Da der Künstler in das Forschungsprojekt eingebunden ist (mal mehr und mal weniger eng), bildet er sich seinen eigenen Standpunkt in Bezug auf dieses Projekt und kann sich in gewissen Fällen parallel zu den Forschern daran beteiligen. Philippe Leroux formuliert hier in seinen eigenen Worten das Forschungsprojekt von Nicolas Donin und Jacques Theureau, an dem er selbst teilgenommen hat. Er lässt die verschiedenen Formen des Wissens und der Bewußtwerdung Revue passieren, die aus einer Serie von Interviews hervorgingen, für die er sich in den Jahren 2003 2006 zur Verfügung gestellt hatte. Diese Interviews, die sich auf zwei seiner Hauptwerke aus diesen Jahren bezogen, sollten die Entstehungssituation der Komposition rekonstruieren.
Si las consecuencias de la "genéticain vivo" no han sido precisadas todavía a nivel metodológico ni epistemológico, se puede sí, desde ya, iniciar una reflexión sobre uno de los aspectos inéditos de este tipo de estudio: el creador, dado que está necesariamente implicado (más o menos estrechamente) en el proyecto de investigación, construye un punto de vista específico sobre este último, pudiendo incluso, en algunos casos, contribuir con el mismo, paralelamente a los investigadores. Philippe Leroux propone aquí una reformulación en sus propios términos, del proyecto de investigación de Nicolás Donin y Jacques Theureau, en el cual participó, y observa los diferentes tipos de saber o de toma de consciencia ocasionados por la serie de "entrevistas de puesta en situación de composición" a los que se prestó durante un periodo de tres años (2003-2006), relativas a dos de sus principales obras de este periodo.

Estando por precisar nos planos metodológico e epistemológico as consequências da "genética in vivo", é possível começar a reflectir sobre um aspecto inédito desse tipo de estudo: visto que o criador se acha necessariamente implicado (e pouco distante) no projecto de investigação, pode formar uma opinião específica sobre ele e, em certos casos, pode mesmo juntar o seu contributo ao dos investigadores. Philippe Leroux propõe aqui uma reformulação, em termos que lhe são próprios, do projecto de investigação de Nicolas Donin e Jacques Theureau em que participou, e percorre os diferentes tipos de saber ou de tomada de consciência causados pela série de "entrevistas de regresso à situação de composição" a que se entregou por três anos (2003-2006), relativamente a duas das suas principais obras do período.

Anche se le conseguenze della "genetica in vivo" sono ancora da precisare sul piano metodologico e epistemologico, è già ora possibile riflettere su un aspetto inedito di questo tipo di ricerca: poiché il creatore è necessariamente implicato (più o meno strettamente) nel progetto di ricerca, egli sviluppa un punto di vista su se stesso e potrebbe, in certi casi, contribuirvi in parallelo con gli studiosi. Philippe Leroux propone qui una riformulazione in termini propri del progetto di ricerca di Nicolas Donin e Jacques Theureau al quale ha partecipato; ripercorre così i diversi tipi di sapere o di presa di coscienza sviluppati dalla serie di "incontri di contestualizzazione della composizione" ai quali si è dedicato nel corso di tre anni (2003-2006), svolti attorno a due delle sue principali opere di questo periodo. 